Der Einfluss von ökologisch-ethischer Vermögensverwaltung auf Unternehmen

\section{Verantwortlich investieren wirkt}

\author{
Unternehmen werden seit Mitte der 70er Jahre von verschiedenen Seiten auf \\ ihr ökologisches und soziales Verhalten untersucht und dabei entweder mittel- \\ bar oder unmittelbar aufgefordert, sich zu verbessern. Ökologisch-ethische \\ Bewertungen für die Vermögensverwaltung spielen hier eine immer wichtigere \\ Rolle.
}

$\mathrm{L}$ Von Thomas Loew ange Jahre hat der Markt für ökologischethische Finanzanlagen ein Schattendasein geführt. Die in den 80er und Anfang der 90er Jahre angebotenen ,grünen“ Investmentfonds investierten in der Regel in Ökonischenpioniere wie zum Beispiel Body Shop oder in Unternehmen aus ökologisch unbedenklichen Branchen, wie Fahrradherstellung oder Umwelttechnik. Der Markt geriet in Bewegung, als 1999 der Dow Jones Sustainability Index aufgelegt wurde. Durch eine rechnerische Rückwärtsbetrachtung des Index für den Zeitraum von 1996-99 wurde demonstriert, dass Konzerne mit überdurchschnittlichen ökologischen und sozialen Leistungen auch eine bessere Aktienperformance erzielen (1).

In der Folge wurden zahlreiche Finanzanlageprodukte mit dem Ziel entwickelt, eine gute bis überdurchschnittliche Rentabilität unter Berücksichtigung von ethischen Anforderungen anzubieten. Seitdem ist der Markt für ökologisch-ethisches Investieren, englisch socially responsible investment (SRI), erheblich gewachsen und entsprechend häufiger werden große Unternehmen auf ihr Umwelt- und Sozialverhalten analysiert. Für die Sustainable-Governance-Forschung stellt sich nun die Frage, inwieweit sich der SRI-Markt auf das Verhalten der Unternehmen auswirkt.

Anhand einer kürzlich veröffentlichten Studie des deutschen Aktieninstituts und der European Business School (EBS) kann der Wirkungsmechanismus zumindest teilweise nachvollzogen werden (2). In der Untersuchung geben 96 börsennotierte Unternehmen, davon 24 aus dem DAX-30, Auskunft über ihre Einschätzungen zu nachhaltigkeitsorientierter Unternehmensfiuhrung und SRI. Die Fragestellungen können überwiegend folgenden Kategorien zugeordnet werden: (i) Motivatoren für Nachhaltigkeit und SRI, (ii) (resultierende) Zielsetzung und (iii) Steuerungswirkung von SRI.

\section{- Motivation}

Das Interesse die Unternehmensführung stärker an Nachhaltigkeitsanforderungen auszurichten, basiert auf unterschiedlichen Überlegungen. Insgesamt werden mehrheitlich positive Zusammenhänge zwischen einer guten Umwelt- und Sozialperformance und einem guten generellen Management einerseits und einer besseren Aktienperformance andererseits gesehen.

Es wird allerdings auch deutlich, dass ökologische und soziale Aktivitäten in der Regel von der Gewinnsituation abhängen. Überdurchschnittliche Leistungen im Nachhaltigkeitsmanagement werden von Unternehmen eher erbracht, wenn hohe Gewinne erwirtschaftet werden. Aus Unternehmenssicht stellen diese Aktivitäten also strategisch sinnvolle, aber in wirtschaftlich schwierigen Phasen keine notwendigen Investitionen dar.

\section{Ziel Anerkennung}

Anleger können diesen Zusammenhang nutzen, indem die Nachhaltigkeitsleistungen als Indikator für die wirtschaftliche Situation des Unternehmens verwendet werden. Dabei wird angenommen, dass durch überdurchschnittliche ökologische und soziale Leistungen strategische Vorteile erschlossen werden. Das Geld ist also gut investiert, was Ende der 90er Jahre durch die überdurchschnittliche (rechnerische) Performance der Nachhaltigkeitsindizes bestätigt werden konnte.

Diese Überlegungen werden zumindest von einem Teil der großen Unternehmen nachvollzogen. So streben etwas die Hälfte der Befragten, dabei 18 der 24 DAX-30 Unternehmen, die Aufnahme in SRI-Indizes oder SRI-Fonds an, da ihnen diese in den Bereichen Umweltschutz und Soziales ein überdurchschnittliches und damit strategisch weitsichtiges Verhalten sichtbar bestätigen. Je größer und bekannter ein Unternehmen ist, desto wichtiger ist diese externe Anerkennung.
SRI-Fonds und -Indizes legen unterschiedliche Auswahlverfahren zugrunde. Für große Unternehmen kommen nur solche Indizes und Fonds in Betracht, die wie SAM und FTSE4good ein ,,best of class"-Verfahren verwenden, also innerhalb einer Branche die Vorreiter identifizieren. Bei Indizes und Fonds, die wie der Natur-Aktien-Index NAX ihren Fokus auf Ökonischenpioniere oder Umwelttechnologien legen, können die großen Unternehmen die zugrunde gelegten Auswahlkriterien durchgängig nicht erfüllen, weshalb sich diese Fonds und Indizes für die Konzerne nicht zur Differenzierung eignen.

\section{Steuerungswirkung}

Wenngleich die Aufnahme in die best-of-classbasierten Indizes von großen börsennotierten Unternehmen angestrebt wird, orientieren sie sich nicht eng an den Kriterien des Auswahlverfahrens. Gut ein Drittel der Befragten gab an, dass die Fragebögen der Nachhaltigkeitsanalysten lediglich Hinweise für die Gestaltung des Nachhaltigkeitsmanagements liefern. Ein weiteres Drittel verwendet die Fragebögen überhaupt nicht. Die Unternehmen entwickeln demnach eigene Strategien, wobei die Fragebogenanforderungen eine eingeschränkte Rolle spielen.

Die hier vorgenommene Auswertung der Untersuchung des Deutschen Aktieninstituts und der EBS macht in Bezug auf Sustainable Governance deutlich,

- dass die best-of-class basierten Indizes und Fonds bei den großen börsennotierten Unternehmen einen gewissen Wettbewerb in Bezug auf das Nachhaltigkeitsmanagement auslösen und

- dass die Anforderungen der Fonds und Indizes den Unternehmen zwar Anregungen für die Gestaltung ihres Nachhaltigkeitsmanagements geben, somit einen leichten aber keinen starken Einfluss ausüben.

\section{Aktive Einflussnahme der Investoren}

Eine weitere SRI-Strategie ist die aktive Einflussnahme (active engagement) der Anleger auf die Unternehmen, in die sie investiert haben. Eine active-engagement-Strategie wird traditionell von großen institutionellen Investoren umgesetzt, da sie aufgrund ihrer großen Anlagevolumina Einfluss ausüben können. Ausschlaggebend für die Motivation von Pensionskassen und vergleichbar großen institutionellen Anlegern ist nach Schumacher der quasi-ewige Anlagehorizont und die Schwierigkeit, aus großen Engage- 
ments kurzfristig auszusteigen (3). Im Sinne ,if you can't sell you must care" besteht in einer aktiven Einflussnahme, zunächst unabhängig ob sie SRI-bezogen ist oder nicht, ein wichtiges Potenzial, den langfristigen Kurswert der Aktien im eigenen Portfolio zu sichern. Diese aktive Einflussnahme auch auf ökologisch-ethische Aspekte auszuweiten liegt nahe, wenn der oben dargestellte Zusammenhang zwischen nachhaltiger Unternehmensführung und shareholder value zugrunde gelegt wird. Verschiedene Regulierungen, wie die gesetzliche Verpflichtung zur Stimmrechtsausübung in den USA aber auch die britische Offenlegungspflicht, fördern active engagement in Bezug auf ökologisch-ethische Fragestellungen.

Einen Eindruck über den möglichen Umfang und die Professionalität einer active-engagement-Strategie vermittelt der britische Vermögensverwalter Insight Investment, der mit einem verwalteten Volumen von 67 Milliarden Britischen Pfund (95 Milliarden Euro) zu den größten britischen Vermögensverwaltern zählt. In einer eigenen Abteilung werden Unternehmen gezielt auf Schwachpunkte in ihrer ökologischen und sozialen Performance untersucht und Kampagnen entwickelt. Beispielsweise ist Insight im zweiten Quartal 2003 in folgenden Bereichen aktiv gewesen (4):

- Verantwortliches Supply Chain Management: Sieben Unternehmen wurden angesprochen, um sie zur Einhaltung von internationalen Standards in ihrer Wertschöpfungskette zu bewegen.

- Menschenrechte: 74 Unternehmen wurden schriftlich aufgefordert, ihr Verhalten in Bezug auf die Einhaltung der Menschenrechte zu überprïfen.

- Burma: Nachdem die burmesische Regimekritikerin Aung San Suu Kyi erneut unter Hausarrest gesetzt wurde, befragte Insight den BAT-Konzern erneut zu seinen Geschäften in Burma.

- OECD-Guidelines: Mit Vertretern von Anglo American und Rio Tinto wurden Gespräche über die Umsetzung der OECD-Guidelines geführt.

Um Einfluss auf Unternehmen auszuüben, schreibt Insight Briefe an den Vorstand, führt Benchmarkings zu best practice durch oder führt direkte Gespräche mit Vertretern des jeweiligen Unternehmens. Ergänzend erstellt Insight Studien, in denen zum Beispiel der Zusammenhang zwischen Corporate Governance und Corporate Social Responsibility (CSR) untersucht wird und setzt sich auch auf politischer Ebene für eine bessere Umsetzung bereits ver- einbarter Standards ein. Inzwischen bietet Insight seine engagement-Aktivitäten auch Dritten als Dienstleitung an.

\section{Fazit}

Zusammenfassend ist festzustellen, dass im Wesentlichen die zwei hier betrachteten SRI- Anlagepolitiken Einfluss auf das Nachhaltigkeitsmanagement von großen börsennotierten Unternehmen ausüben. Die best-in-class-Auswahlverfahren und das active engagement. Welcher Einfluss größer ist, kann nur vorsichtig abgeschätzt werden. Es scheint plausibel, dass die gezielten Aktivitäten im Rahmen von active engagement eher zu unmittelbaren Reaktionen seitens der Unternehmen führen. Demgegenüber ist unklar, wie stark und auf wie viele Unternehmen die neu geschaffene Wettbewerbssituation wirkt, die durch best-practice-Auswahlverfahren ausgelöst wird. Schließlich sind beide SRI-Strategien im Kontext des Engagements von umweltorientierten und sozialen NGOs zu sehen. Werden diese Kampagnen von den SRIAnalysten als berechtigt angesehen, dürften sie diese bei ihrer Arbeit berïcksichtigen und somit als Verstärker für die Stimme der NGOs wirken.

\section{Anmerkungen}

(1) Loew, Thomas: Nachhaltig Investieren. In: Ökologisches Wirtschaften 2/2003, sowie allgemein den Themenschwerpunkt "Perspektiven nachhaltiger Finanzanlagen“ der Ausgabe 2/2003.

(2) Deutsches Aktieninstitut, Institut für Ökologie und Unternehmensführung (Hrsg.): Nachhaltigkeit und Shareholder Value aus Sicht börsennotierter Unternehmen. Studien des deutschen Aktieninstituts, Heft 22, Frankfurt 2003.

(3) Schumacher-Hummel, Ingeborg: Mit Pensionen grüne Kasse machen. Einflussmöglichkeiten institutioneller Anleger auf nachhaltige Unternehmensführung. In: Ökologisches Wirtschaften 2/2003, S. 22-23.

(4) Insight Investment (Hrsg.): Investor Responsibility Bulletin, London 2003, S. 3.

\section{Der Autor}

Thomas Loew ist wissenschaftlicher Mitarbeiter im Forschungsfeld Ökologische Unternehmenspolitik am Institut für ökologische Wirtschaftsforschung (IÖW).

Kontakt: IÖW, Potsdamer Str. 105, 10785 Berlin, Tel. 030-88459417, E-Mail: Thomas.Loew@ioew.de
SOZIALEユTECHNIK 然

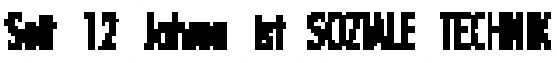

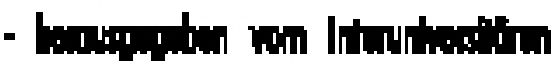

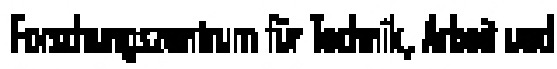

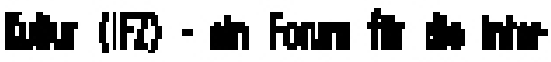

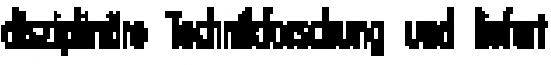

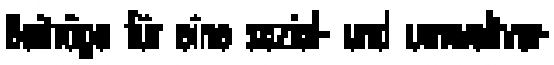
trojlkho Gustatung von Iecino-

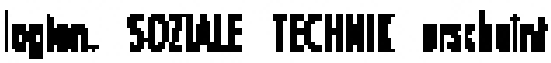

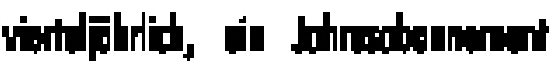

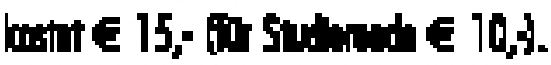

Kostenloses Probeabonnement!

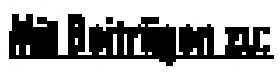

口 Technologie \& Politik

口 Umwelt \& Energie

- lleve Biotechnologien

口 Frauen \& Technik

- Informations-\&

Kommunikationstechnologien

SOZIALE

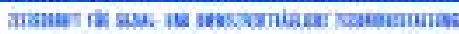




(c) 20I0 Authors; licensee IÖW and oekom verlag. This is an article distributed under the terms of the Creative Commons Attribution Non-Commercial No Derivates License (http://creativecommons.org/licenses/by-nc-nd/3.o/), which permits unrestricted use, distribution, and reproduction in any medium, provided the original work is properly cited. 